\title{
Propagation Measurements during Daytime for RazakSAT S-band Space to Earth Satellite Signal Transmission
}

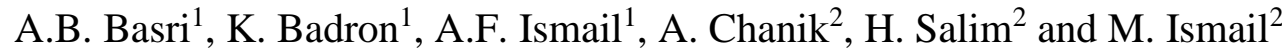 \\ ${ }^{1}$ Department of Electrical and Computer Engineering, Kulliyyah of Engineering, \\ International Islamic University Malaysia (IIUM), \\ Jln. Gombak, Selangor, Malaysia \\ ${ }^{2}$ National Space Agency (ANGKASA), Malaysia Space Centre \\ 42700 Banting, Selangor \\ atikahbalqis32@gmail.com, khairayu@iium.edu.my,af_ismail@iium.edu.my \\ abadi@angkasa.gov.my,hamid@angkasa.gov.mymaszlan@angkasa.gov.my
}

\begin{abstract}
Adequate fade margins for all conditions are critical in ensuring reliable satellite operation. The required fade margin value for specific desired quality of service (QoS) can be established from the statistics of outage due to attenuation. In the case of clear sky attenuation, the value is much dependent on the atmospheric layer conditions and their compositions. For absolute Free Space, the signal loss is only dependent on distance and frequency. In this study, the effects of distance were analysed in the investigation of identifying the most appropriate clear sky attenuation values during daytime. The RazakSAT received signal levels were matched according to the distances between the satellite and the Ground station. Clear sky conditions were confirmed using S-band (Terminal Doppler Weather Radar) TDWR reflectivity information. With this value, the satellite operator can decide execution of power uplink and mitigation technique if necessary. The RazakSAT S-band $(2.232 \mathrm{GHz})$ transmission signal data of were furnished by Malaysian National Space Agency (ANGKASA) and radar data were acquired from Malaysian Meteorology department (MMD). The findings offer awareness of required fade margin as distance varies during each flight path. The collected data will also be employed in the investigation of Free Space Path Loss (FSPL) formulation applicable for satellite link in tropical region.
\end{abstract}

Keywords: Atmospheric layer; FSPL; Reflectivity; TDWR

\section{Introduction}

Satellite communication is one of the most significant and beneficial technologies that has been ever developed. Many commercial and private communication services can be instantly offered by satellite communication, for example the voice, data transfer and video services. This technology has great appeal to many business applications, since it can be easily deployed in the shortest possible time and in select instances, with minimal investment [1]. The two basic components of a satellite communications system are the space segment and the ground segment. The space segment typically consists of the spacecraft and the launch mechanism while the ground segment on the other hand, conventionally comprises of the Earth station with a network control centre for the entire satellite system operation [2]. Free Space Path Loss (FSPL) or any loss determination is an important aspect to consider before the launching of any satellite. This is due to the fact that once the satellite is in orbit, limited modification to the space segment hardware can be carried out and will definitely involve a very high cost. The ionosphere is a region

Received (August 10, 2017), Review Result (November 15, 2017), Accepted (November 26, 2017) 
composed of free electrons and positive ions in the atmosphere. The existence of charged particles in the ionosphere itself brings a great influence on the electrical properties within the region, thus makes a possibility for communicating over far distances. The ionosphere layers situated between 100 and $400 \mathrm{~km}$ above the Earth's surface are shown in Figure 1 below. There is a possibility occurrence of signals reflection and such may interrupt the signal propagation. The communication over far distances between nations is achieved by multiple hops between the ionosphere and the Earth's surface [3]. FSPL is the loss in signal strength of an electromagnetic wave that would be resulted from a line-of-sight path through free space with no obstacle to cause reflection or diffraction. Theoretically, the free-space path loss equation is proportional to the square of the distance between the transmitter and receiver, and also proportional to the square of the frequency of the radio signal. For ease of calculation, the formulation can also be expressed in decibel as shown in Equation 1.

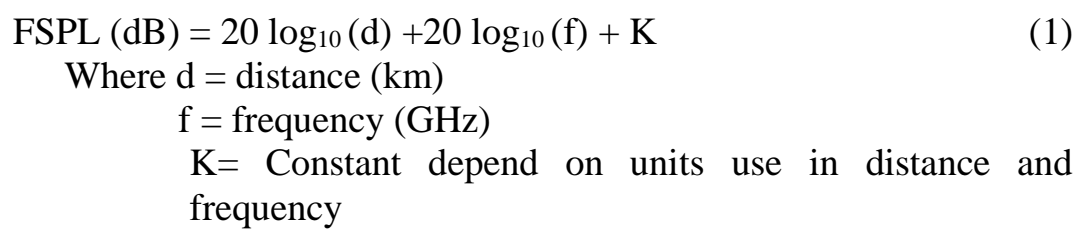

In this analysis, the effect of distance on the RazakSAT satellite X-band signal propagation will be quantified. The experiment aims at a coordinated acquisition of propagation measurement across several ground stations located in tropical region.

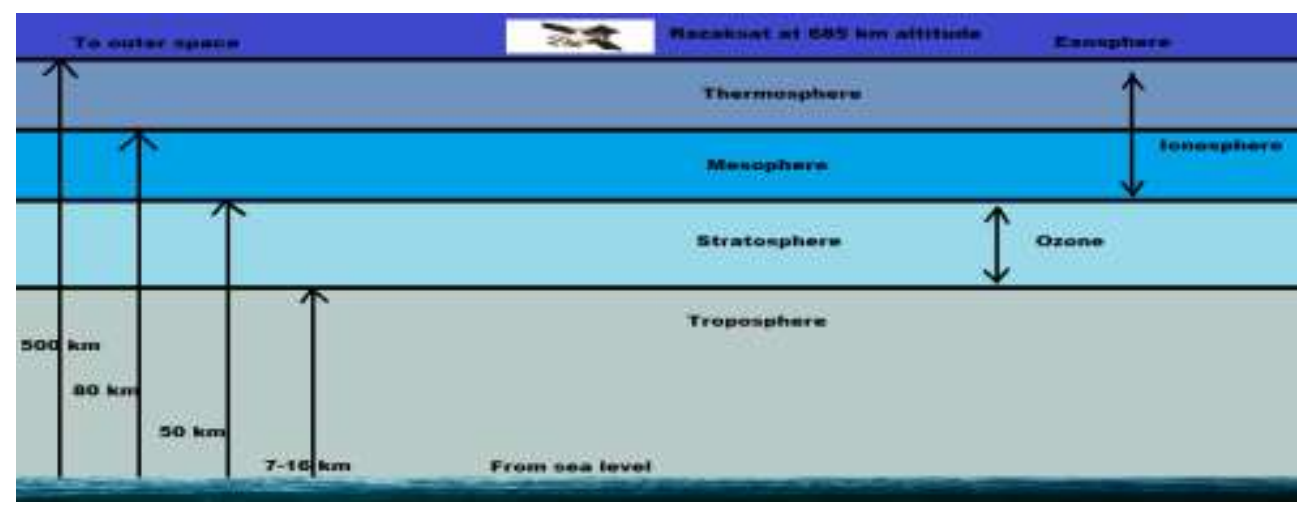

Figure 1. Major Layer of the Earth's Atmosphere

Clear sky condition is regarded when there is absence of precipitation. The condition does not produce serious signal amplitude fading as when compared to instances with presence of precipitation. However, even during clear sky condition, the variation of signal strength due to distance travel during space propagation even for system operating at frequencies below $10 \mathrm{GHz}$ still require quantification. The measured and predicted values of signal loss can be used as critical parameters in the link budget design where transmit power control system can be deployed.

\section{Description of System and Data}

\subsection{RazakSAT Satellite System}

The RazakSAT satellite was launched into the Near Equatorial Orbit (NEqO) on July 15 2009. Its orbital distance from Earth surface is at about $685 \mathrm{~km}$ altitude, also commonly referred to as the exosphere layer of atmosphere. With $7^{\circ}$ to $9^{\circ}$ inclination from its geographical location, RazakSAT has abundant imaging opportunities to cover 
equatorial regions. The satellite weight is only about $200 \mathrm{~kg}$ and hence considered as a small or medium size satellite. The satellite is a high-resolution remote sensing satellite equipped with a pushbroom camera, encompassing one panchromatic and four multispectral detectors. RazakSAT is operated and controlled from the ground segment located in Sungai Lang, Banting, Malaysia. The S-band $(2.232 \mathrm{GHz})$ transmission signals from RazakSAT were monitored and tracked by a Hexapod antenna receiver. The high-speed image transmission system is capable of downloading up to $32 \mathrm{Gbits}$ of stored image data within 3 to 4 daytime passes [4]. The specifications of the RazakSAT satellite are listed in the Table 1 below. The uplink carriers and downlink carrier between satellite and earth station can observed in Figure 2.

Table 1. RazakSAT Common Specification

\begin{tabular}{|c|c|c|c|}
\hline \multicolumn{3}{|l|}{ Item } & System specifications \\
\hline Orbit & & & $685 \mathrm{~km}$ at $\mathrm{NEqO}$ \\
\hline Altitude & & & $7^{\circ}-9^{\circ}$ \\
\hline Ground & Sampling & Distance & Four multi-spectral: $5 \mathrm{~m}$ \\
\hline (GSD) & & & Panchromatic: $2.5 \mathrm{~m}$ \\
\hline Mass & & & $187.6 \mathrm{~kg}$ \\
\hline Power & & & $>300 \mathrm{~W}$ \\
\hline Data dov & link & & $30 \mathrm{Mbps}$ ( X-band) \\
\hline Payload & & & Medium-sized aperture camera \\
\hline Short rev & sits & & Six times a day over Malaysian region \\
\hline Image pr & duction & & Level 1 Product within 5 hours from reception \\
\hline
\end{tabular}

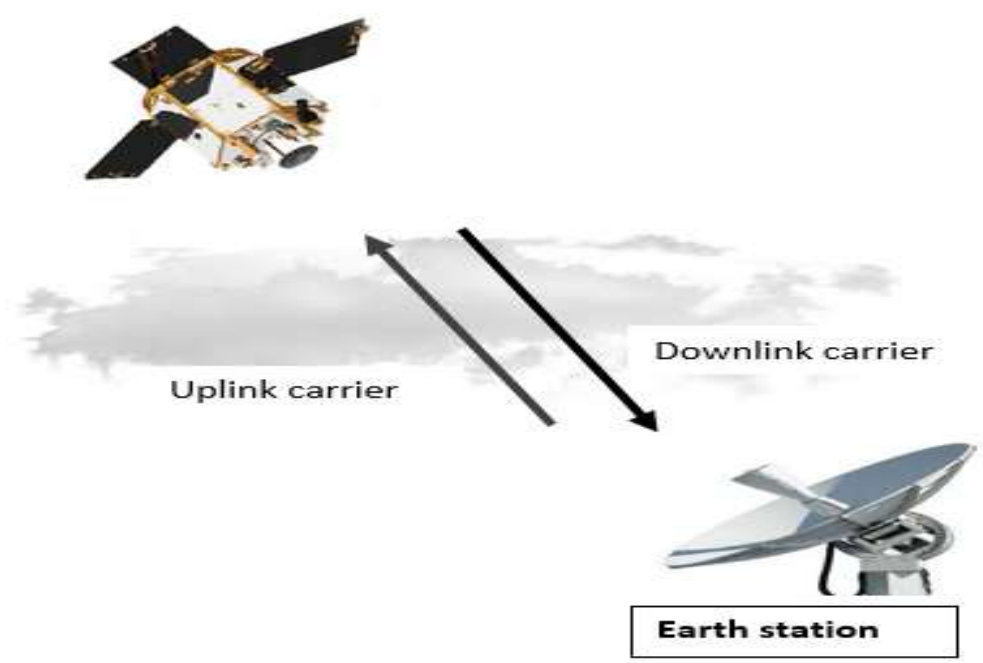

Figure 2. Schematic of Satellite Uplink and Downlink System

\subsection{Radar System}

The corresponding radar data was acquired from the Malaysian Meteorological Department's (MMD). MMD is the authorized department in preparing the Radar data throughout Malaysia. These data are raw data and special software is needed to generate required information. A terminal Doppler weather radar (TDWR) was installed in the area about $10 \mathrm{~km}$ north of Kuala Lumpur International Airport (KLIA). The S-band TWDR centre frequency is $2.75 \mathrm{GHz}$. As for the analysis of atmospheric effects during clear sky, data based on single polarization was exploited. The radar system is set to work in two scanning modes. Each mode incorporates specific sweeps of the surrounding area at a predetermined elevation 
angles. The antenna for this radar rotates at the two revolution per minute (RPM) during clear conditions. Its pulse repetition frequency (PRF) is equal to $0.3 \mathrm{KHz}$ and the scanning covers elevation angles of less than $5^{\circ}$. The radar revolves at faster speed of four revolution per minute (RPM) with PRF equals to $1 \mathrm{KHz}$ for angles of elevation in between $5^{\circ}$ to $40^{\circ}$ when rain event is detected. The radar has the coverage capability of up to $120 \mathrm{~km}$ radius [5].

\subsection{Data Collection}

The 3-D position of RazakSAT satellite orbiting the Earth at every second was determined and actual displacement from the ground station at any given time can be calculated. A typical flight path of the RazakSAT satellite orbiting the Earth is as shown in Figure 3. The analysis pertaining to received signal level was correlated against the distance that signals propagate from current locations of RazakSAT satellite to the ground station. Literally, the position of any orbiting satellite is considered from the ground station point of view. The position is typically defined by Azimuth (Az) and Elevation $(0 \varepsilon)$ angles. In the case of azimuth, it is defined as the angle in the direction the satellite, measured in the horizon plane from geographical north in right-handed or clockwise direction. The azimuth is basically the range from $0^{\circ}$ to $360^{\circ}$. On the other hand, the angle between a satellite and the observer's (ground station's) horizon plane is called elevation angle. The elevation would range from $0^{\circ}$ to $90^{\circ}[6]$.

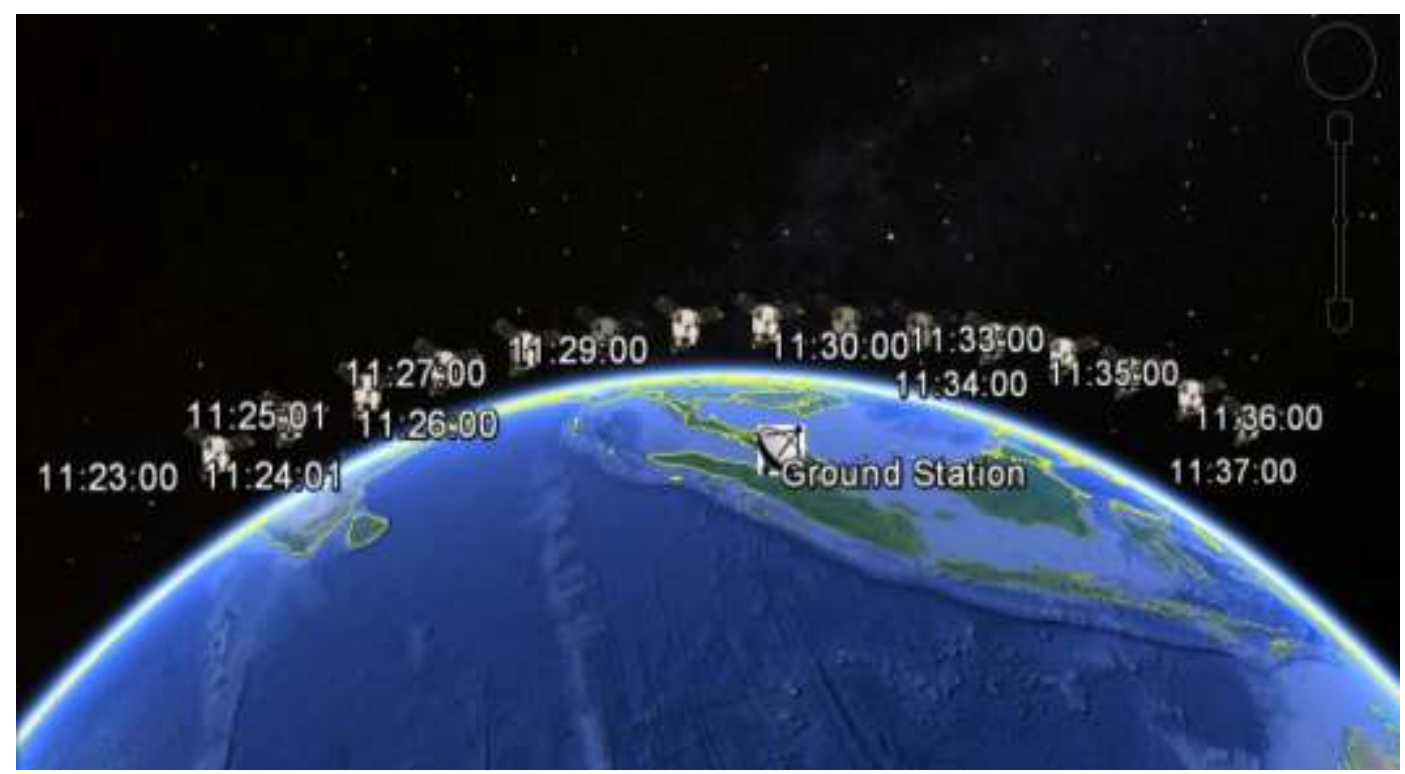

Figure 3. RazakSAT Satellites Motion

\subsection{Distance Calculation}

The positional data received from RazakSAT were used to compute the distance displacement. From the current location of the RazakSAT satellite, azimuth and elevation angles from the perspective of the ground station can be determined. The distance formula Equation (2) for non-geostationary satellite orbit was employed to calculate the distance between two points which is the shortest distance over two points [7]. The formula is made available by ITU-R S.1257 recommendation where analytical method involving elevation value can be used to compute visibility statistics for non-geostationary satellite orbit satellites as seen from a point on the Earth's surface [8]. 


$$
\mathrm{d}_{\mathrm{N}}=\sqrt{r^{2} \sin ^{2} \varepsilon+2 r h+h^{2}}-r \sin \varepsilon
$$

where:

$\varepsilon$ : Elevation angle of a satellite from a station

$r:$ Earth radius $=6376 \mathrm{~km}$

$h$ : Altitude of the non-geostationary satellite orbit satellite $(\mathrm{km})$

$\mathrm{d}_{\mathrm{N}}$ : Distance to the non-geostationary satellite orbit satellite from a station $(\mathrm{km})$

The RazakSAT data were used concurrently in the determination of distance between the satellite to the ground station and monitoring of the received power signal levels. Shown in Figure 4 below is the location of the RazakSAT satellite during 16 July 2009 at 11:30:00 am. By using the formula above, the calculated distance is $693.8441 \mathrm{~km}$ from the ground station. While, Figure 5 demonstrated the location of the RazakSAT's satellite during 16 July 2009 at 11:24:01 am. The distance is about of $2626.68 \mathrm{~km}$ to the ground station.
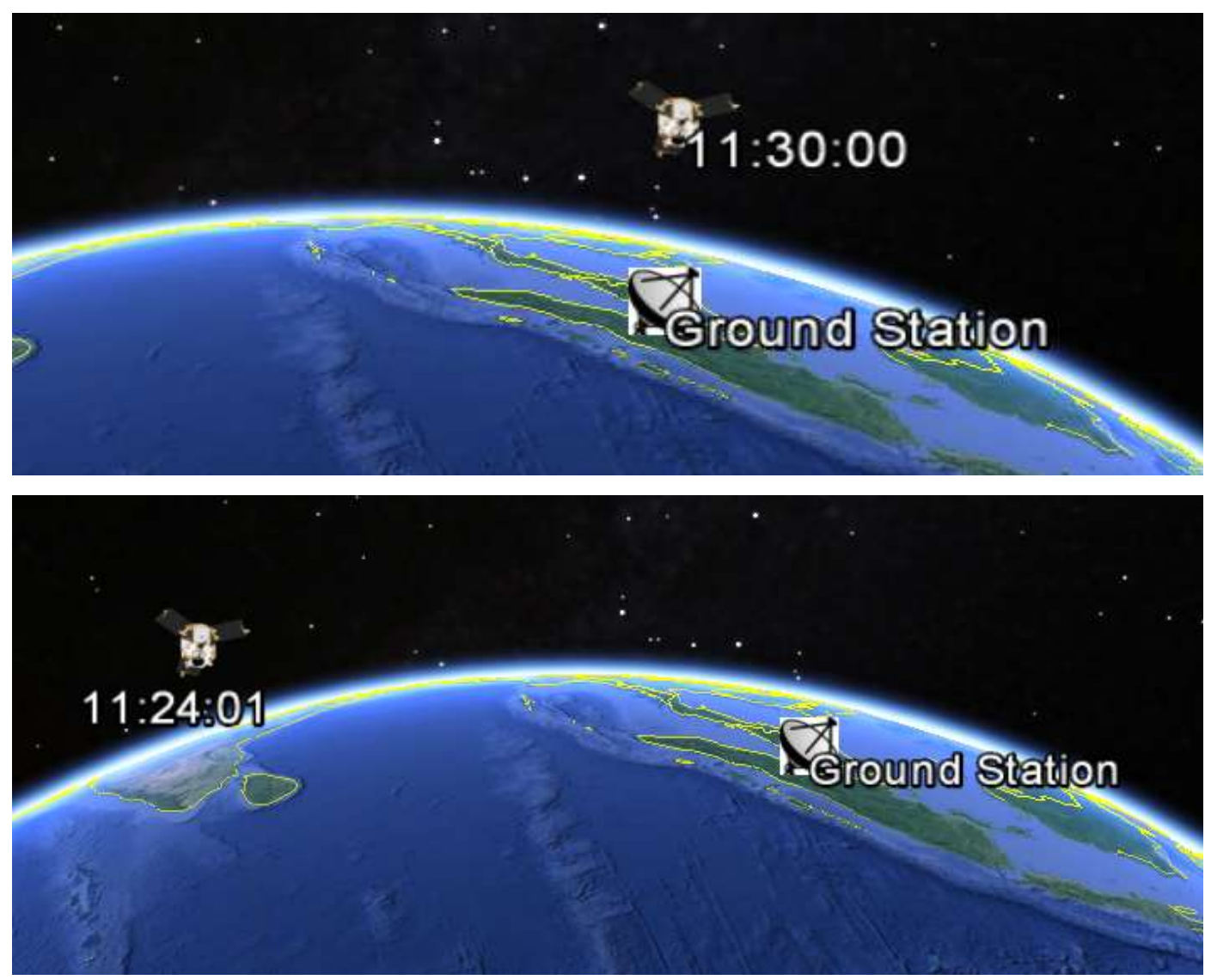

Figure 5. Location of RazakSAT at 11:24:01

\section{Results and Discussion}

In this section, the result was presented for (i) radar analysis and (ii) statistical analysis. Fourteen revisits of RazakSAT data which in total of 11200 of information set had being examined and grouped together in order to explore the locations of the RazakSAT satellite for every second based on their latitude and longitude. With location information, the elevation angles were determined and distance 
computations were executed. For every displacement point, the level of each received signal originated from RazakSAT satellite was examined.

(i) Radar analysis

The radar information was used in this analysis in order to verify and substantiate claim of clear sky condition. By using radar display software the Iris Vaisala, the reflectivity value can be established and the clear sky condition can be corroborated. The constant altitude plane position indicator (CAPPI) view above the ground station during RazakSAT satellite flight path is shown in Figure 6. According to the ITU-R recommendation P.839 the estimated typical rain height for Malaysia is at about $4.5 \mathrm{~km}$. The display of the desired Iris product output was used to check existence of any reflectivity value. Clear sky can be assumed for conditions where reflectivity value (dBZ) less than 5 [9]. The display of rain-height indicator (RHI) in Figure 7 confirms that the condition is clear sky where of no rain and no cloud is present during the process when the RazakSAT was transmitting data to the ground station.

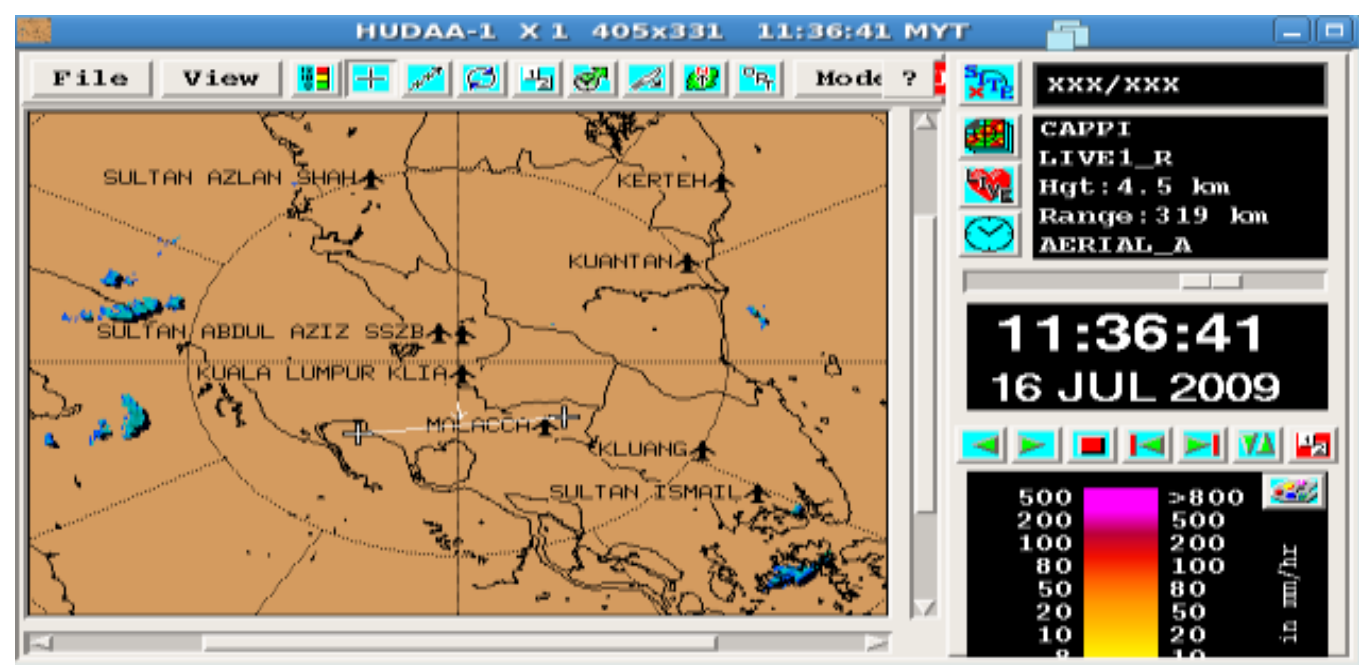

Figure 6. CAPPI view above the Ground Station

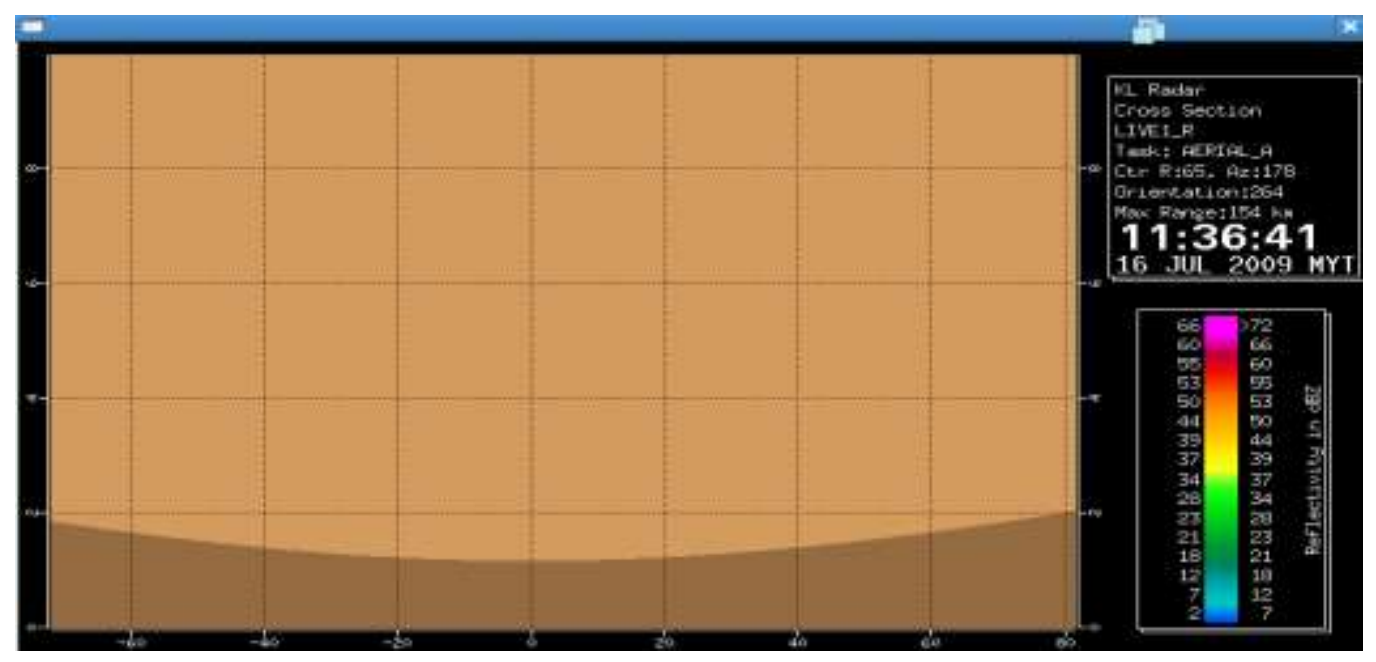

Figure 7. RHI view above Ground Station 


\section{(ii) Statistical analysis}

In this section, the highlighted result involves the RazakSAT received signals recorded during a time period of one visit that took place at 11:20:47 until 11:37:22 on 16 July 2009. From the data set, the graph for distance against time was plotted and labelled as Figure 8.A graph for received power signal against time was also generated as shown in Figure 9. The received signal and distance were then tabulated together in one graph. Best-fit plot was produced based on the tabulated data and the relevant correlation coefficients were listed in Table 2. As can be observed in Figure 10, the best-fit plot suggests that higher attenuation values can be observed at distances nearest to the ground station. As the distance gets farther away, the attenuation value reduces accordingly. The variation of signal strength was indeed influenced by the propagation path between the transmitter and the receiver.

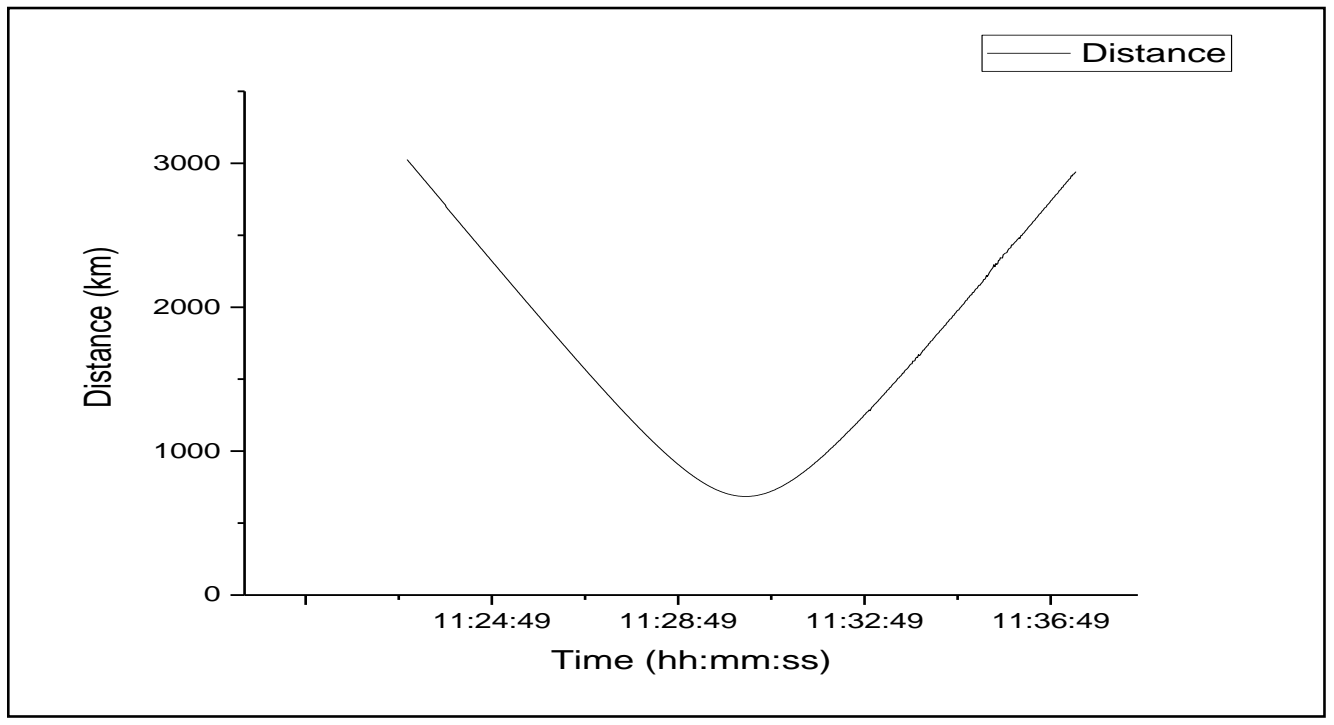

Figure 8. Graph Distance against Time

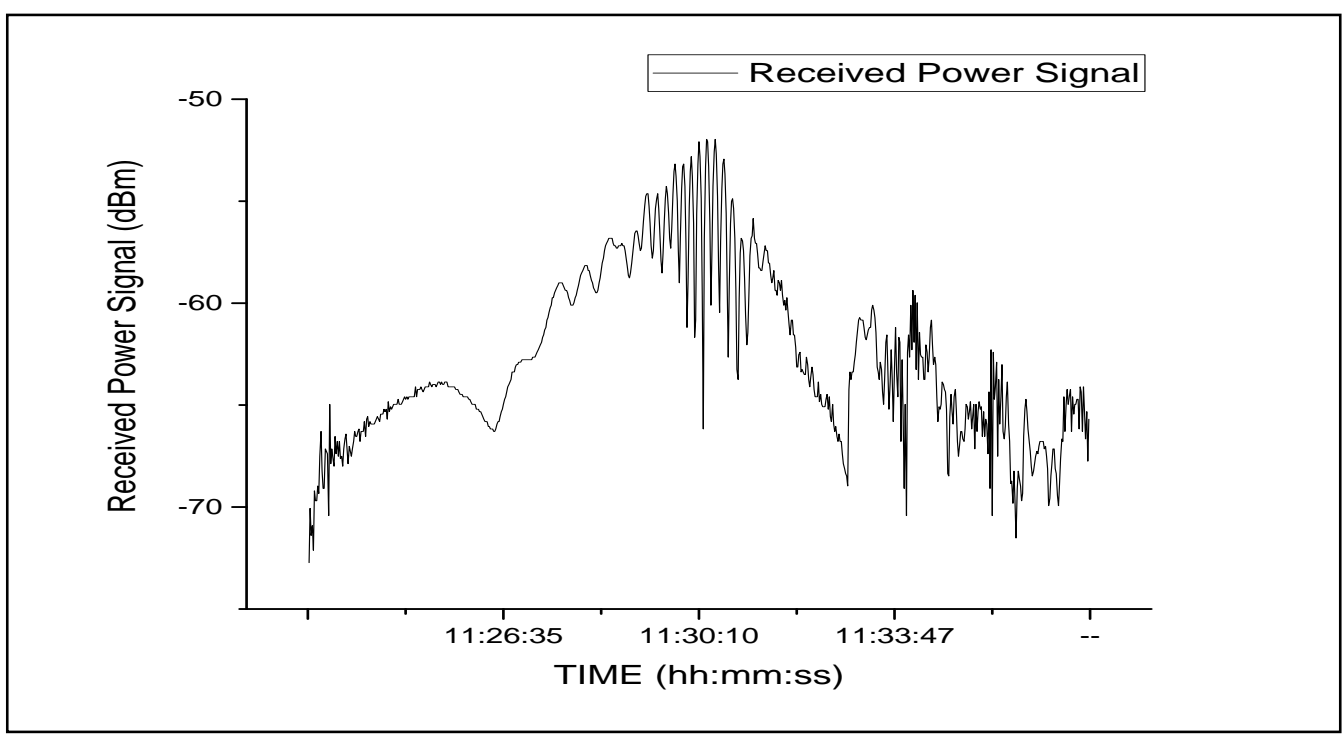

Figure 9. Power Signal Values against Time 


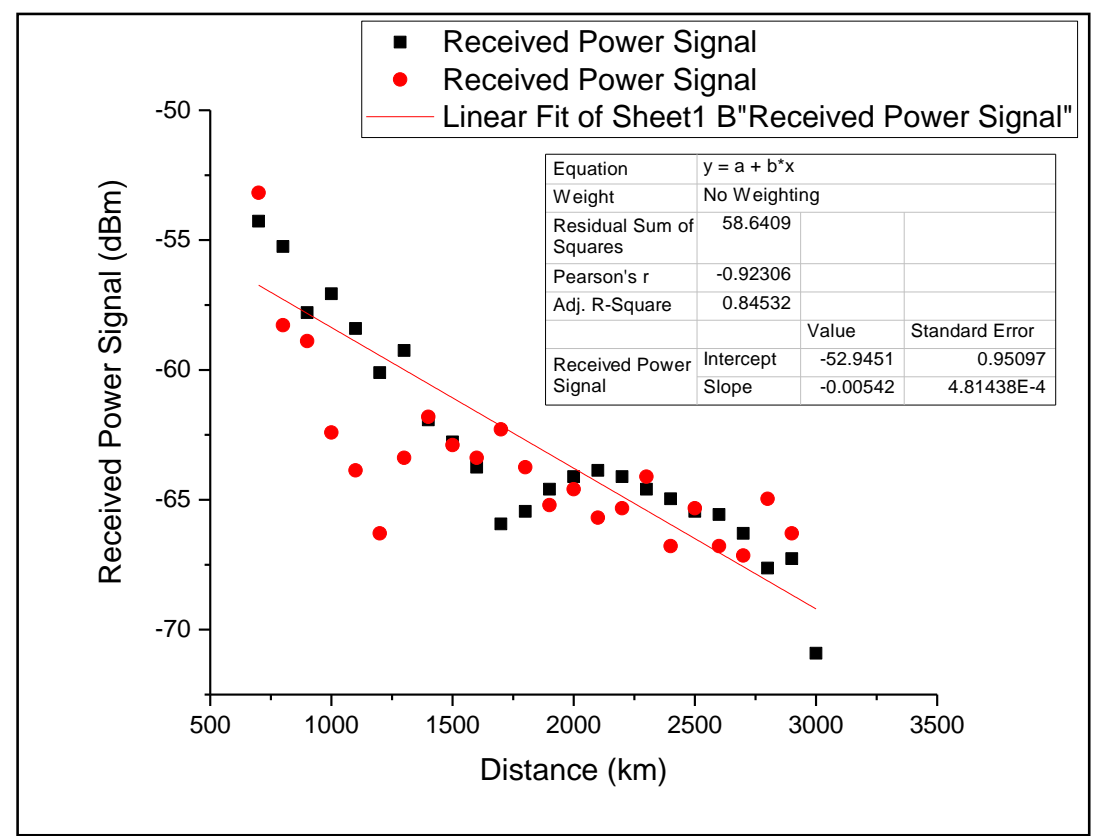

Figure 10. Best-Fit Plot for Received signal and Distance

The best-fit plot established the Equation 2,

$$
y=-62.9451 x-0.00542
$$

Table 2. Coefficients

\begin{tabular}{|c|c|c|}
\hline Coefficients & Symbols & Values \\
\hline Slope coefficient & $\mathrm{m}$ & -62.9451 \\
\hline Slope intercept coefficient & $\mathrm{C}$ & -0.00542 \\
\hline
\end{tabular}

\section{Conclusion}

From the investigation of the RazakSAT satellite received power signals, the correlation that associate attenuation level with distance travelled by signal was identified. In general, the notion that the father away the signal propagate, the higher the attenuation level it will experience. With the help of radar information, the findings also indicate that the attenuation value in clear sky is not in a continuous constant state and solely affected by the distance travelled. During clear sky, even when there is no rain or clear indication of hydrometeors; as confirmed using the radar information, signals are still get attenuated by other elements. The assessment suggests fade margin that is totally based on FPSL might not be the best option. Reduction of signal amplitude is by no doubt overwhelm dictated by distance travelled but further analyses are required in order to comprehensively quantify factors affecting signal propagation, System operator should carefully consider the effects other distance in order to achieve the prescribed Quality of Services (QOS) of any specific services. It is hoped that all affecting factors will be quantified and a revised free space path loss formulation for satellite link operating in tropical region can be derived. 


\section{Acknowledgement}

The authors would like to acknowledge the University for the Support all the way through the research. The Malaysian Ministry of Higher Education is gratefully acknowledged for funding. This research is being sponsored under Fundamental Research Grant Scheme research project in generation of new theories, concepts and ideas.

\section{References}

[1] "Analytical Method to Calculate Visibility Statistics for NGSO Satellites as Seen from a Point on the Earth's Surface”, Recommendation ITU-R S.1257, International Telecommunication Union, Geneva, (1997).

[2] K. Badron, A. F. Ismail, J. Din and A. R. Tharek, "Rain induced attenuation studies for V-band satellite communication in tropical region", Journal of Atmospheric and Solar-Terrestrial Physics, vol. 73, no. 5, (2011), pp. 601-610.

[3] S. Cakaj, B. Kamo, A. Lala and A. Rakipi, "The coverage analysis for low earth orbiting satellites at low elevation", Int. J. Adv. Comput. Sci. Appl(ijacsa), vol. 5, no. 6, (2014).

[4] J. H. Deifel and A. J. Pena, An Analysis of CONUS Based Deployment of Pseudolites for Positioning, Navigation and Timing (PNT) Systems", (No. AFIT-ENV-MS-15-S-37). Air Force Institute Of Technology Wright-Patterson Alb Oh Graduate School Of Engineering And Management, (2015).

[5] M. Drusch, U. Del Bello, S. Carlier, O. Colin, V. Fernandez, F.Gascon and A. Meygret, "Sentinel-2: ESA's optical high-resolution mission for GMES operational services", Remote Sensing of Environment, Text Book, vol. 120, (2012), pp. 25-36.2.

[6] B. J. Kim, S. Park, E. E. Kim, H. S. Chang, W. Park, J. Seon and A. Sabirin-Arshad, "MACSAT-A Mini-Satellite Approach to High Resolution Space Imaging", (2003).

[7] G. Larssen. "Ice Detection and Tracking Based on Satellite and Radar Images", (2015).

[8] J. William, Martin, and A. Shapiro, "Discrimination of bird and insect radar echoes in clear air using high-resolution radars", Journal of Atmospheric and Oceanic Technology, vol. 24, no. 7, (2007), pp. 1215-1230.

[9] J. Sun, and N. Andrew Crook, "Dynamical and microphysical retrieval from Doppler radar observations using a cloud model and its adjoint. Part II: Retrieval experiments of an observed Florida convective storm", Journal of the Atmospheric Sciences, vol. 55, no. 5, (1998), pp. 835-852.

Authors

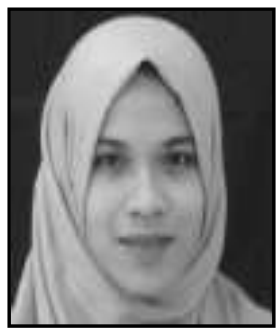

A. B. Basri, she graduated in 2014 B. Eng and 2017 MSc in Communication from the International Islamic University Malaysia (IIUM). She is currently a research officer whilst pursuing her Ph.D studies at the Electrical and Computer Engineering Department, IIUM. Her research interests are in radar radiometry and wireless communication.

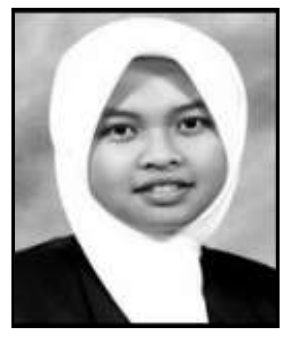

K. Badron, she obtained her BEng (2006), Msc (2011) and Ph.D (2016) from International Islamic University Malaysia (IIUM). She is currently one of the faculty members of Faculty of Engineering, IIUM and recently commenced her research areas in Radar and Radiometry research, quantifying propagation effects on microwave and millimetre wave links. Khairayu is a member of IEEE, IEM, BEM and MTSFB. She has published and co-authored more than 15 papers in International Journals as well as Conferences on subjects relating to rain attenuation in the tropical regions. 


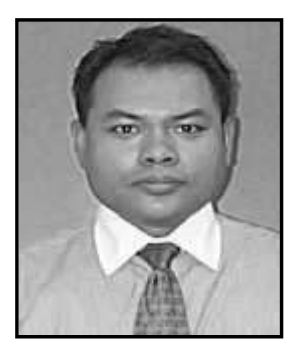

A.F. Ismail, he is a currently a lecturer at Department of Electrical and Computer Engineering, Faculty of Engineering, International Islamic University Malaysia (IIUM). He completed his bachelor degree studies in Electrical Engineering at Gannon University, Pennsylvania, USA with Cum Laude Latin honors. He holds MSc and PhD from University of Essex, UK and University of Bath, UK, respectively. His research interests include development of active and passive target tracking algorithms, radio frequency and wireless systems and microwave and milimetrewave technology. He is registered with Board of Engineering Malaysia as a Professional Engineer and also a senior member of IEEE.

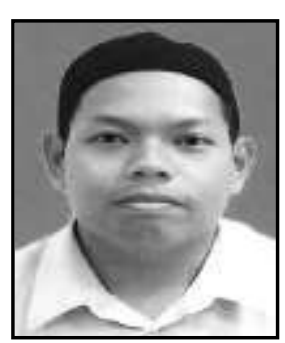

Abadi Chanik, he gained the B.E. degree in electronic engineering from the Universiti Malaysia Sarawak, Malaysia, in 2005. He is currently awaiting the approval for his $\mathrm{PhD}$ from the University of Surrey in the field of spacecraft control. He is a member of the Board of Engineers (BEM) Malaysia and since 2006, he is a Research Officer with the Malaysia Space Agency (ANGKASA). His work includes the operation of the Malaysian and other satellites from neighboring country specifically in the ground segments. His research interest includes development of a ground facility for spacecraft testing and its earth segment.

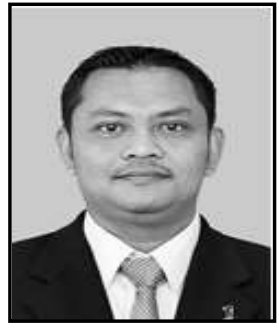

Hamid Salim, he is currently a research officer at Agensi Angkasa Negara (ANGKASA), Ministry of Science,Technology and Innovation (MOSTI). He completed his Bachelor Degree studies in Kolej Universiti Teknologi Tun Hussein Onn (KUiTTHO). He holds MSc from Universiti Teknologi MARA (UiTM) majoring in Telecommunication and Information Engineering. He involve in the R\&D activity in space development for the low cost mission including RazakSAT.

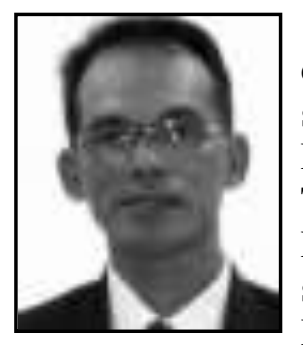

M. Ismail, he is the Malaysian National Space Agency director. He directs the satellite ground operation as well as the spacecraft testing facilities. He was the principal engineer for Malaysian Remote Sensing Satellites -RazakSAT and TiungSAT. Maszlan received his BEng and MSc from the National University of Malaysia specializing in wireless and satellite communication. He is one of the committee members of IEEE Malaysian chapter and Asia-Pacific Regional Space Agency Forum. 